\title{
Opportunities in Angularly Resolved Dark-field STEM using Pixelated Detectors.
}

Lewys Jones ${ }^{1}$, Hao Yang ${ }^{1}$, Katherine E. MacArthur ${ }^{1}$, Henning Ryll ${ }^{2}$, Martin Simson ${ }^{3}$, Heike Soltau ${ }^{3}$, Yukihito Kondo ${ }^{4}$, Ryusuke Sagawa ${ }^{4}$, Hiroyuki Banba ${ }^{4}$, and Peter D. Nellist ${ }^{1}$.

1. Department of Materials, University of Oxford, Oxford, UK

2. PNSensor GmbH, Otto-Hahn-Ring 6, 81739 München, Germany

3. PNDetector GmbH, Sckellstraße 3, 81667 München, Germany

4. JEOL Ltd.,3-1-2 Musashino Akishima Tokyo 196-8558 Japan

Pixelated sensors in the detector plane of the scanning transmission electron microscope (STEM) offer many opportunities for extracting valuable information from the bright-field (BF) disk including synthesising annular bright-field images, differential phase-contrast images, and ptychographic phase reconstruction [1]. However, by reducing microscope camera-length, these pixelated detectors can also be used to record the scattered electron flux outside the BF disk. This scattering has previously been recorded using annular dark-field (ADF) detectors however there the operator must choose what annular range the DF signal is to be integrated over at the point of the imaging and this can limit operational flexibility. Some instruments offer two or more annular DF detectors to record more than one angular range but in this case the ratio of collection angles of these two detectors is fixed. Use of two detectors allows low-angle or medium-angle (LAADF and MAADF) images to be recorded which have been found to yield useful strain or structural information [2], [3].

Here we demonstrate the first lattice resolution ADF data recorded using a pixelated detector. We show how various types of DF images can be synthesised including LAADF, MAADF and high-angle (HAADF) images. Moreover, the angularly resolved data recording allows for a shift from a situation where a user must choose from one of a limited set of available detector collection angles, to a regime where the required detector conditions can be chosen after the experiment.

Figure 1 shows the readout from a pnCCD (S)TEM camera, a direct electron pixelated detector from PNDetector (264x264 pixels in this case), mounted on a probe corrected JEOL ARM200-CF. Owing to the massive difference in intensity inside and outside of the BF disk a beam-blanker was used to block the BF disk. Figure 1 also shows an enlargement showing the detection of individual electron scattering events at high angles meaning this approach also offers a route to direct quantification of the ADF signal without the need to normalise for detector sensitivity [4]. An ADF image was first focussed using a conventional detector with a PtCo nanoparticle as a test object (Figure 2). After focussing, the physical $\mathrm{ADF}$ detector was retracted and a 256x256 real-space probe array dataset recorded at a rate of 1000 detector plane frames per second (total scan time 66s). After the experiment, the imaging mode was selected in post-processing. First to view the overall data-quality the whole ADF signal was integrated (Figure 2, top). This data confirms that the magnification and resolution were sufficient to achieve atomic resolution, and that the scanning stability was sufficient at the relatively long $1 \mathrm{~ms}$ dwell-time. This image also shows the position of a twin-boundary on the left side of the particle, indicated by the pair of red arrows. Using the 4D data-set scattering ranges were selected for integration including LAADF (25.8-26.7 mrad) and MAADF (30.1-32.7 mrad), Figure 2 bottom. In these plots the area to the left of the twin-boundary appears first light, then dark respectively. 
In conclusion, we have shown that use of a pixelated detector in the diffraction plane of the STEM allows for recording of a full 4D scattering data-set. Post-processing of this data allows for various imaging modes to be synthesised after the experiment itself. Tuning of the annular ranges of the virtual detectors allows specific contrast to be extracted around crystallographic, composition or strain features that may be washed out by larger physical ADF detectors [5].

\section{References:}

[1] T. J. Pennycook et al, Ultramicroscopy in-press (2014).

[2] S. Hillyard and J. Silcox, Ultramicroscopy 58 (1995), p. 6-17.

[3] G. Zhu et al., Appl. Phys. Lett. 105 (2014), p. 231607.

[4] J. M. Lebeau and S. Stemmer, Ultramicroscopy 108 (2008), p. 1653-8.

[5] This work was supported by the EU grant 312483 - ESTEEM2 and EPSRC grant EP/K040375/1.

The authors thank Johnson Matthey for the PtCo sample and for financially supporting KEM.

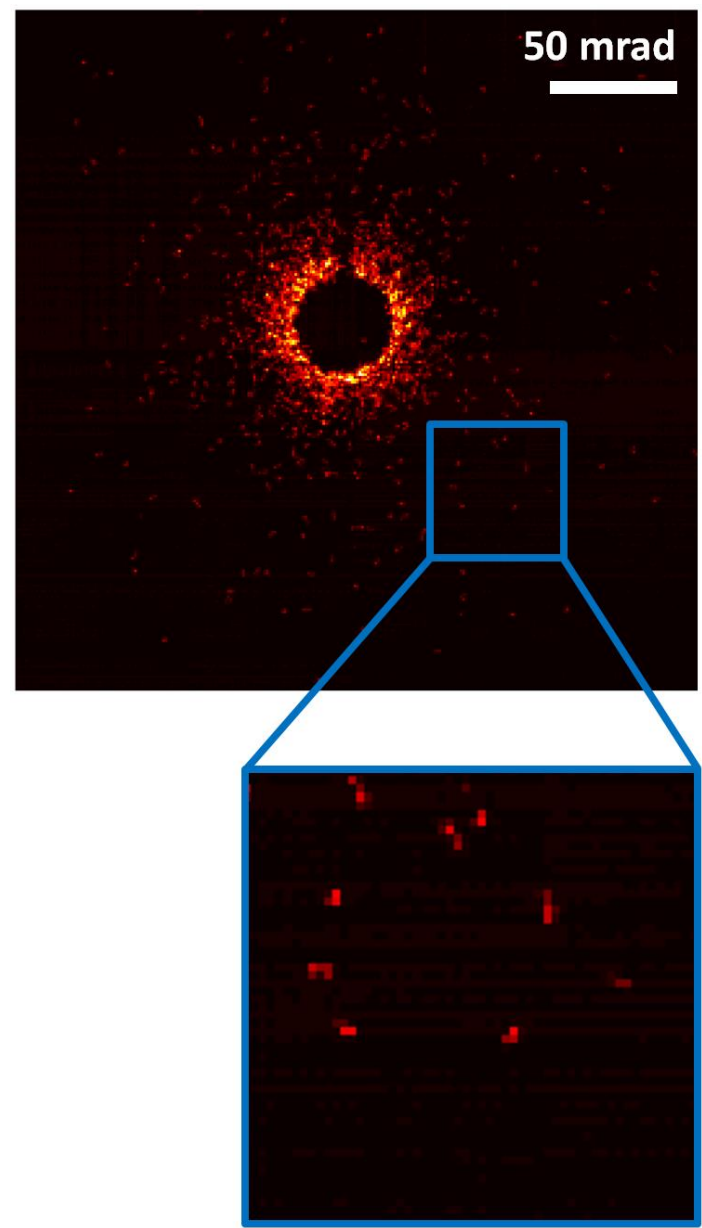

Figure 1. pnCCD (S)TEM camera readout for an example real-space probe position. A beamstopper was used to mask the intense BF-disk. Enlargement shows the sensitivity of the detector to individual electron scattering events.
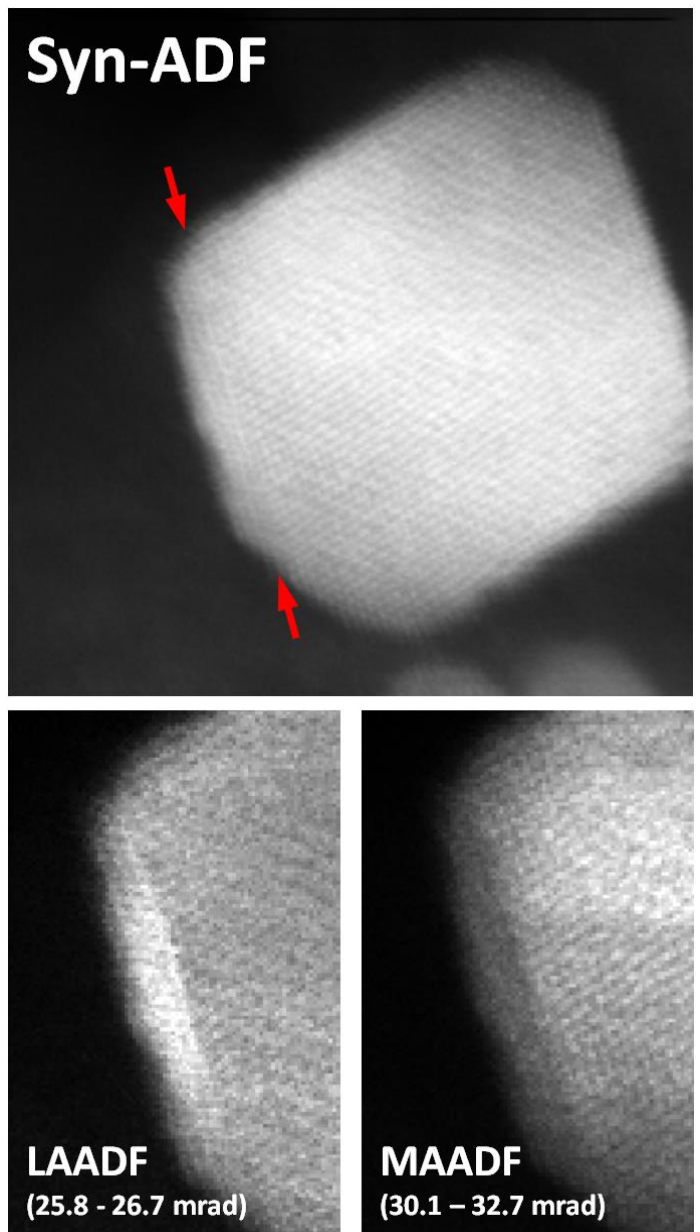

Figure 2. Example images synthesised from the 4D dataset. Top panel shows the integrated ADF signal. Arrows indicate the position of a twinboundary. Lower panels show synthetic low-angle and medium-angle ADF images where the particle edge region appears bright and dark respectively. 